\title{
Synthesis and Antibacterial Activity of Novel Schiff Bases of Thiosemicarbazone Derivatives With Adamantane Moiety
}

Jiahui Zhu

Changchun University of Technology

Guosheng Teng

Changchun University of Technology

Dongfeng Li

Changchun University of Technology

Ruibin Hou

Changchun University of Technology

Yan Xia ( $\sim$ hmz20130521@163.com)

Changchun University of Technology https://orcid.org/0000-0002-4974-7266

\section{Research Article}

Keywords: thiosemicarbazone, antibacterial, adamantane, MIC

Posted Date: April 14th, 2021

DOl: https://doi.org/10.21203/rs.3.rs-407353/v1

License: (9) This work is licensed under a Creative Commons Attribution 4.0 International License. Read Full License 


\section{Abstract}

Increased bacterial resistance to antibiotics is a major threat to human health, and it is particularly important to develop novel antibiotic drugs. Here, we designed a series of Schiff base thiosemicarbazone derivatives containing an adamantane moiety, and carried out structural characterization of the compounds and in vitro antibacterial activity tests. Compound $7 \mathbf{e}$ was as effective as the commonly-used antibiotic ampicillin against the Gram-negative bacterium Escherichia coli, and compound $\mathbf{7 g}$ had a good inhibitory effect against Gram-positive Bacillus subtilis. These findings provide data for the development of better thiosemicarbazone antibacterial agents.

\section{Introduction}

In the past 30 years, only limited progress has been made in research into antibacterial drugs with new mechanisms and core structures [1-3]. Amoxicillin, norfloxacin, and ciprofloxacin are the commonest drugs used to treat bacterial infection, but are associated with severe side effects. Toxicity and bacterial resistance to the drugs play an important role in the failure of treatment $[4,5]$. The development of antibacterial drugs with novel structures is very important research for clinical application [6].

Recently, considerable attention has been focused on substituted thiosemicarbazone derivatives because of their interesting biological activities. Compounds with a thiosemicarbazone structure are known to possess tranquilizing, muscle relaxing, psychoanaleptic, hypnotic, ulcerogenic, antidepressant, antibacterial, antifungal, analgesic, and anti-inflammatory properties [7-14]. Thiosemicarbazones are a type of Schiff base formed by condensation of thiosemicarbazide and an aldehyde or ketone. Studies have shown that changing the structure of the aldehydes and ketones or introducing different active groups on position $\mathrm{N}(4)$ can improve the antibacterial and anticancer activities of the compounds [15]. Therefore, synthesis of thiosemicarbazone compounds with various structures and study of their structure-activity relationships have important theoretical significance and potential practical application value.

Adamantane compounds have shown relatively good antivirus, antitumor, and anti-Parkinson's syndrome activities [16-21]. The introduction of adamantyl groups into the molecular structure of other compounds often enhances the biological activity of the compound. The adamantyl group has relatively good fat solubility, so can greatly increase the membrane permeability of a compound [22-25]. Much research has been carried out on thiosemicarbazone derivatives, but no work has been done screening adamantyl thiosemicarbazone derivatives for their antibacterial activity.

In this paper, novel adamantyl thiosemicarbazone derivatives were synthesized by the condensation of an adamantyl phenyl aldehyde with a thiosemicarbazide. The structures of the compounds were elucidated by infrared (IR), ${ }^{1} \mathrm{H}$ NMR, and ${ }^{13} \mathrm{C}$ NMR spectroscopies, and mass spectrometry. The activities of the compounds were screened in vitro against Bacillus subtilis (a Gram-positive bacterium) and 
Escherichia coli (Gram-negative). To the best of our knowledge, this is the first report that thiosemicarbazone analogues having an adamantane moiety inhibit the growth of bacteria.

\section{Results And Discussion}

\section{Chemistry}

Scheme 1 shows the procedure for synthesis of novel Schiff base derivatives of thiosemicarbazones with an adamantane moiety (compounds $7 \mathbf{a}-\mathbf{h}$ ). The Friedel-Crafts alkylation reaction of bromoadamantane and toluene in the presence of anhydrous potassium carbonate and palladium carbon was used to obtain 1-( $p$-toluene)adamantane (2). Compound 2 underwent free radical substitution reaction using NBS and BPO to yield benzyl bromide (3), followed by oxidation to give 4 [4-(1-adamantyl)benzaldehyde]. The aromatic amines $\mathbf{5} \mathbf{a}-\mathbf{h}$ were used to prepare the thiotoluamides $\mathbf{6} \mathbf{a}-\mathbf{h}$ in basic conditions. Finally, $\mathbf{4}$ was reacted with $\mathbf{6 a}-\mathbf{h}$ to produce thiosemicarbazone Schiff base derivatives $7 \mathbf{a}-\mathbf{h}$.

The yields of all thiosemicarbazone products were $62-86 \%$. The compounds obtained were stable in both the solid and solution states. Analytical data for these compounds were consistent with their composition. The structures of thiosemicarbazone compounds 7a-h were confirmed on the basis of infrared (IR), ${ }^{1} \mathrm{H}$ nuclear magnetic resonance (NMR), ${ }^{13} \mathrm{C}$ NMR, and electrospray ionization mass spectral (ESI/MS) data, which showed their negative ion peaks $[\mathrm{M}+\mathrm{H}]^{+}$. In the infrared (IR) spectra, $\mathrm{N}-\mathrm{H}$, aromatic and aromatic $\mathrm{C}-\mathrm{Hs}, \mathrm{C}=\mathrm{N}, \mathrm{C}=\mathrm{C}, \mathrm{C}-\mathrm{N}$ and $\mathrm{C}-\mathrm{O}$ bands stretching vibrations were observed. $\mathrm{N}-\mathrm{H}$ bands belonging to the thiosemicarbazone group were recorded around $3250 \mathrm{~cm}^{-1}$. Aromatic and aliphatic $\mathrm{C}-\mathrm{H}$ stretching bands appeared between $3190 \mathrm{~cm}^{-1}$ and $2910 \mathrm{~cm}^{-1} . \mathrm{C}=\mathrm{N}$ and $\mathrm{C}=\mathrm{C}$ groups gave rise to the bands in the region $1600-1442 \mathrm{~cm}^{-1}$. C-N and C-O bands were observed in the region $1287-1124 \mathrm{~cm}^{-1}$. In the ${ }^{1} \mathrm{H}$-NMR spectra, adamantyl group protons had peaks between $1.74 \mathrm{ppm}$ and $2.07 \mathrm{ppm}$. The chemical shift values of aromatic ring protons were between $6.92 \mathrm{ppm}$ and $8.37 \mathrm{ppm}$, depending on the substituent groups. The characteristic azomethine $(-\mathrm{CH}=\mathrm{N}-)$ protons appeared between $6.77 \mathrm{ppm}$ and $7.22 \mathrm{ppm}$. Besides, the characteristic $\mathrm{N}-\mathrm{H}$ protons were seen at $9.93-11.91 \mathrm{ppm}$. In the ${ }^{13} \mathrm{C}-\mathrm{NMR}$ spectra, aliphatic and aromatic carbons were observed between 21.05-56.56 ppm and 113.78-163.19 ppm, respectively. the azomethine $(-\mathrm{CH}=\mathrm{N}-)$ and the thiocarbamoyl carbons were detected at 143.06-143.93 ppm and 175.10-177.60 ppm, respectively. In Mass (MS) analysis, the mass spectral data were coherent with their molecular formulas.

\section{Antimicrobial activity}

The antibacterial activity of the synthesized compounds against B. subtilis (Gram-positive) and E. coli (Gram-negative) was evaluated using the disk inhibition method and the microdilution method to determine MICs [26-28]. Ampicillin was used as a positive control drug. The results are shown in Tables 1 and 2.

In the disk inhibition method (Table 1), a diameter of the inhibition zone is $20 \mathrm{~mm}$ or more indicates that the drug has a very strong antibacterial effect on the strain; the inhibition zone of $10-20 \mathrm{~mm}$ is 
categorized as strong antibacterial effect; a diameter of 5-10 mm indicates a moderate antibacterial effect; and diameter of $5 \mathrm{~mm}$ or less indicates that there is little or no antibacterial effect of the compound on the strain [29].

Based on the growth inhibition response, the antibacterial inhibition of compounds $7 \mathbf{a}$ and $7 \mathbf{g}$ on $B$. subtilis with concentration of $8 \mu \mathrm{g} / \mathrm{mL}(22 \mathrm{~mm})$ had very strong activity and other concentrations (10-20 $\mathrm{mm}$ ) including strong activity. The antibacterial inhibition of compounds $7 \mathbf{d}$ and $7 \mathrm{~h}$ on $B$. subtilis with concentrations of $1-8 \mu \mathrm{g} / \mathrm{mL}(10-20 \mathrm{~mm})$ had strong activity, while compounds $7 \mathrm{c}$ and $7 \mathrm{e}$ with concentrations of $2-8 \mu \mathrm{g} / \mathrm{mL}(10-20 \mathrm{~mm})$ indicated strong activity. The antibacterial inhibition of compounds $7 \mathbf{b}$ and $7 f$ on $B$. subtilis with concentrations of $1-8 \mu \mathrm{g} / \mathrm{mL}(5-10 \mathrm{~mm})$ had moderate activity. The antibacterial inhibition of compound $7 \mathrm{a}$ and $7 \mathbf{e}$ on $E$. coli with concentrations of $1-8 \mu \mathrm{g} / \mathrm{mL}$ $(10-20 \mathrm{~mm})$ had the best inhibitory effect, similar to the effect of positive control Ampicillin. Meanwhile compounds $7 \mathrm{~b}-\mathrm{c}$ and $\mathbf{7 g}-\mathrm{h}$ with concentrations of $1-8 \mu \mathrm{g} / \mathrm{mL}(10-20 \mathrm{~mm})$ had strong activity against $E$. coli, but compounds $7 f$ with concentrations of $1-2 \mu \mathrm{g} / \mathrm{mL}(5-10 \mathrm{~mm})$ including moderate activity and concentrates of $4-8 \mu \mathrm{g} / \mathrm{mL}(10-20 \mathrm{~mm})$ are categorized as strong activity.

Table 1

Antibacterial activity of compounds $7 \mathrm{a}-\mathrm{h}$ at different concentration

\begin{tabular}{|c|c|c|c|c|c|c|c|c|}
\hline \multirow[t]{3}{*}{ Comp. } & \multicolumn{8}{|c|}{ Diameter of inhibition zone of different strains(mm) } \\
\hline & \multicolumn{4}{|c|}{ Escherichia coli $(\mu \mathrm{g} / \mathrm{mL})$} & \multicolumn{4}{|c|}{ Bacillus subtilis $(\mu \mathrm{g} / \mathrm{mL})$} \\
\hline & 1 & 2 & 4 & 8 & 1 & 2 & 4 & 8 \\
\hline $7 a$ & 12 & 13 & 15 & 17 & 15 & 16 & 19 & 20 \\
\hline $7 b$ & 10 & 11 & 13 & 14 & 6 & 7 & 8 & 9 \\
\hline 7c & 11 & 13 & 14 & 15 & 8 & 10 & 11 & 13 \\
\hline 7d & 12 & 13 & 14 & 16 & 10 & 11 & 13 & 14 \\
\hline $7 e$ & 13 & 14 & 15 & 16 & 9 & 10 & 11 & 12 \\
\hline $7 f$ & 8 & 9 & 10 & 11 & 7 & 8 & 9 & 10 \\
\hline $7 g$ & 10 & 12 & 13 & 14 & 17 & 18 & 19 & 22 \\
\hline $7 \mathrm{~h}$ & 8 & 10 & 11 & 13 & 10 & 12 & 13 & 15 \\
\hline Ampicillin & 14 & 14.5 & 15 & 16 & 18 & 20 & 21 & 22 \\
\hline DMSO & - & - & - & - & - & - & - & - \\
\hline
\end{tabular}


Table 2

Minimum inhibitory concentrations of

compounds $7 \mathrm{a}-\mathrm{h}(\mu \mathrm{g} / \mathrm{mL})$, mean \pm standard deviation

\begin{tabular}{|lll|}
\hline Comp. & E. coli & B. subtilis \\
\hline 7a & $8 \pm 0.1$ & $8 \pm 0.02$ \\
\hline 7b & $1 \pm 0.3$ & $4 \pm 0.03$ \\
\hline 7c & $1 \pm 0.04$ & $8 \pm 0.3$ \\
\hline 7d & $2 \pm 0.1$ & $4 \pm 0.02$ \\
\hline 7e & $1 \pm 0.2$ & $2 \pm 0.1$ \\
\hline 7f & $2 \pm 0.03$ & $4 \pm 0.2$ \\
\hline 7g & $2 \pm 0.4$ & $1 \pm 0.01$ \\
\hline 7h & $1 \pm 0.02$ & $8 \pm 0.2$ \\
\hline Ampicillin & 50 & 50 \\
\hline
\end{tabular}

The MIC values of the test derivatives indicate that most compounds exhibit good activity against gramnegative bacteria as shown in Table 2. Compounds $7 \mathbf{b}$ - $\mathbf{d}$ bearing methoxy group on the phenyl had MIC 1-2 $\mu \mathrm{g} / \mathrm{mL}$ for $E$. coli and slightly higher MIC for $B$. subtilis. While compound 7c bearing no substituent had MIC $8 \mu \mathrm{g} / \mathrm{mL}$ for $E$. coli and B. subtilis. Meanwhile, derivatives 7e-g with electron-withdrawing group had MIC $1-2 \mu \mathrm{g} / \mathrm{mL}$ for $E$. coli and almost similar MIC for B. subtilis. Compound $7 \mathrm{~h}$ bearing methyl group showed good antibacterial with MIC $1 \mu \mathrm{g} / \mathrm{mL}$ for E. coli compared with that of compound 7a. From the MIC values as shown in Table 2, it could be easily inferred in general that with substitution on phenyl of thiosemicarbazone had antibacterial activity toward gram-negative bacteria.

\section{Conclusions}

In summary, we investigated the antibacterial activity of novel adamantyl thiosemicarbazones prepared by the reaction of benzaldehyde adamantane with different amine-substituted thiosemicarbazides. Of particular note, in vitro antibacterial activity tests showed that compound $7 \mathbf{e}$ was as effective against $E$. coli (a Gram-negative bacterium) as ampicillin, while $\mathbf{7 g}$ had good activity against the Gram-positive bacterium $B$. subtilis. These data lay a foundation for development of improved thiosemicarbazone antibacterial agents.

\section{Materials And Methods}

\section{General}

The starting materials which include 4-(1-adamantyl)benzaldehyde was prepared according to procedure described previously and other required chemicals were purchased from different commercial sources 
and used without purification unless otherwise stated. The progress of reactions was monitored by using thin layer chromatography (TLC) with silica gel 60 aluminium-backed plates and the ${ }^{1} \mathrm{H}$ NMR and ${ }^{13} \mathrm{C}$ NMR spectra were recorded via Bruker spectrometer $400 \mathrm{MHz}$ as dilute solutions in suitable deuterated solvent at $25^{\circ} \mathrm{C}$. The chemical shifts were recorded on the $\delta$-scale $(\mathrm{ppm})$ using residual solvents as an internal standard (DMSO; ${ }^{1} \mathrm{H} 2.50$, and $\mathrm{CHCl}_{3} ;{ }^{1} \mathrm{H} 7.26$,). Coupling constant were calculated in $\mathrm{Hertz}(\mathrm{Hz})$ and multiplicities were labelled as s (singlet), $d$ (doublet), $t$ (triplet), $q$ (quartet), quint (quintet) and the prefixes br (broad) or app (apparent) were used. IR spectra were recorded on a Specord 2000 spectrometer. Mass spectra were taken on the AB Sciex 6500 QTAP mass spectrometer. Melting points of synthesized compounds were determined by means of a Stuart ${ }^{\text {TM }}$ melting point SMP3 apparatus.

\section{Chemistry}

\section{General procedure for synthesis of derivatives $6 a-h$}

The anilines $(1.0 \mathrm{mmol})$ was dissolved in $20 \mathrm{~mL}$ of DMF, and $\mathrm{NaOH}(1.2 \mathrm{mmol})$ and $\mathrm{CS}_{2}(1.0 \mathrm{mmol})$ were added. After reaction at room temperature for $2 \mathrm{~h}$, hydrazine hydrate $(3.0 \mathrm{mmol})$ was added, and the reaction was continued for $1 \mathrm{~h}$. Cool to room temperature, pour the solution into crushed ice, filter with suction, and recrystallize the filter cake with ethanol to obtain intermediates $\mathbf{6 a - h}$.

\section{N-(2-Fluorophenyl)hydrazinecarbothioamide (6a)}

${ }^{1} \mathrm{H}$ NMR $\left(400 \mathrm{MHz}\right.$, DMSO- $\left.d_{6}\right) \delta 9.34(\mathrm{~s}, 1 \mathrm{H}), 8.08(\mathrm{~s}, 1 \mathrm{H}), 7.19(\mathrm{dq}, J=22.4,7.7 \mathrm{~Hz}, 3 \mathrm{H}) ;{ }^{13} \mathrm{C}$ NMR $(100$ MHz, DMSO- $\left.d_{6}\right) \delta 180.44,156.88,154.46,127.82,127.72,127.13,126.39,126.32,124.16,115.56$; FT-IR $\left(\mathrm{KBr}, \mathrm{cm}^{-1}\right): \mathrm{v}(\mathrm{N}-\mathrm{H}) 3265.31,3193.09, \mathrm{v}(\mathrm{C}-\mathrm{N})$ 1481.12, v(C=S) 1226.07.

\section{N-(2-Methoxyphenyl)hydrazinecarbothioamide (6b)}

${ }^{1} \mathrm{H}$ NMR $\left(400 \mathrm{MHz}, \mathrm{DMSO}-d_{6}\right) \delta 9.94(\mathrm{~s}, 1 \mathrm{H}), 9.18(\mathrm{~s}, 1 \mathrm{H}), 8.74(\mathrm{~s}, 1 \mathrm{H}), 7.04(\mathrm{~s}, 2 \mathrm{H}), 6.90(\mathrm{~s}, 1 \mathrm{H}), 4.83(\mathrm{~s}$, $2 \mathrm{H}), 3.84(\mathrm{~s}, 3 \mathrm{H}) ;{ }^{13} \mathrm{C}$ NMR $\left(100 \mathrm{MHz}\right.$, DMSO- $\left.d_{6}\right) \delta 178.87,149.87,128.63,124.26,121.63,120.16,111.28$, 56.3; FT-IR $\left(\mathrm{KBr}, \mathrm{cm}^{-1}\right): \mathrm{v}(\mathrm{N}-\mathrm{H}) 3313.47,3193.43, \mathrm{v}(\mathrm{C}-\mathrm{N})$ 1457.01, v(C=S) 1238.42.

\section{$\mathrm{N}$-(3-Fluorophenyl)hydrazinecarbothioamide (6c)}

${ }^{1} \mathrm{H}$ NMR $\left(400 \mathrm{MHz}, \mathrm{DMSO}-d_{6}\right) \delta 9.29(\mathrm{~s}, 1 \mathrm{H}), 7.86(\mathrm{~s}, 1 \mathrm{H}), 7.45(\mathrm{~s}, 1 \mathrm{H}), 7.32(\mathrm{~s}, 1 \mathrm{H}), 6.91(\mathrm{~s}, 1 \mathrm{H}) ;{ }^{13} \mathrm{C} \mathrm{NMR}$ $\left(100 \mathrm{MHz}\right.$, DMSO- $\left.d_{6}\right) \delta 179.70,141.72,129.94,119.24,40.29 ; \mathrm{FT}-\mathrm{IR}\left(\mathrm{KBr}, \mathrm{cm}^{-1}\right): \mathrm{v}(\mathrm{N}-\mathrm{H}) 3291.84,3193.52$, $\mathrm{v}(\mathrm{C}-\mathrm{N}) 1456.99, \mathrm{v}(\mathrm{C}=\mathrm{S}) 1224.76$.

\section{N-(3-Methoxyphenyl)hydrazinecarbothioamide (6d)}

${ }^{1} \mathrm{H}$ NMR $\left(400 \mathrm{MHz}, \mathrm{DMSO}-d_{6}\right) \delta 9.14(\mathrm{~s}, 1 \mathrm{H}), 7.49(\mathrm{~s}, 1 \mathrm{H}), 7.20(\mathrm{~d}, J=7.1 \mathrm{~Hz}, 2 \mathrm{H}), 6.67(\mathrm{~d}, J=6.2 \mathrm{~Hz}, 1 \mathrm{H})$, $4.78(\mathrm{~s}, 2 \mathrm{H}), 3.73(\mathrm{~s}, 3 \mathrm{H}) ;{ }^{13} \mathrm{C}$ NMR $\left(100 \mathrm{MHz}\right.$, DMSO- $\left.d_{6}\right) \delta 159.55,140.90,129.28,115.64,109.96,109.21$, 55.54; FT-IR $\left(\mathrm{KBr}^{\mathrm{cm}}{ }^{-1}\right)$ : v(N-H) 3266.26, 3190.51, v(C-N) 1457.42, v(C=S) 1228.66. 
N-Phenylhydrazinecarbothioamide (6e)

${ }^{1} \mathrm{H}$ NMR $\left(400 \mathrm{MHz}, \mathrm{DMSO}-d_{6}\right) \delta 9.11(\mathrm{~s}, 1 \mathrm{H}), 7.66(\mathrm{~s}, 2 \mathrm{H}), 7.42-7.22(\mathrm{~m}, 2 \mathrm{H}), 7.17-7.05(\mathrm{~m}, 1 \mathrm{H}), 4.81(\mathrm{~s}$, $2 \mathrm{H}) ;{ }^{13} \mathrm{C}$ NMR $\left(100 \mathrm{MHz}\right.$, DMSO- $\left.d_{6}\right) \delta 180.05,139.79,128.52,124.49,123.78$; FT-IR $\left(\mathrm{KBr}_{\mathrm{cm}} \mathrm{cm}^{-1}\right) 3300.04$, $3156.12,1635.80,1524.39,1488.93,1282.00,1217.49,1068.07,971.13,894.88,735.19,609.89$.

\section{$\mathrm{N}$-(4-Fluorophenyl)hydrazinecarbothioamide (6f)}

${ }^{1} \mathrm{H}$ NMR (400 MHz, DMSO-d $\left.)_{6}\right) \delta 9.72(\mathrm{~s}, 1 \mathrm{H}), 9.12(\mathrm{~s}, 1 \mathrm{H}), 7.55(\mathrm{~d}, J=37.9 \mathrm{~Hz}, 2 \mathrm{H}), 7.14(\mathrm{dt}, J=17.5,8.8$ $\mathrm{Hz}, 2 \mathrm{H}), 4.78(\mathrm{~s}, 2 \mathrm{H}) ;{ }^{13} \mathrm{C} N M R\left(100 \mathrm{MHz}\right.$, DMSO-d $\left.d_{6}\right) \delta 180.34,136.14,126.33,115.13,114.91$; FT-IR $(\mathrm{KBr}$, $\mathrm{cm}^{-1}$ ) 3307.96, 3108.03, 1636.04, 1493.81, 1284.91, 1208.69, 905.59, 813.48, 502.85.

\section{$\mathrm{N}$-(4-Methoxyphenyl)hydrazinecarbothioamide (6g)}

${ }^{1} \mathrm{H}$ NMR $\left(400 \mathrm{MHz}, \mathrm{DMSO}-d_{6}\right) \delta 9.40(\mathrm{~s}, 1 \mathrm{H}), 8.96(\mathrm{~s}, 1 \mathrm{H}), 7.37(\mathrm{dd}, J=53.0,8.5 \mathrm{~Hz}, 2 \mathrm{H}), 6.88(\mathrm{t}, J=10.1$ $\mathrm{Hz}, 2 \mathrm{H}), 4.70(\mathrm{~s}, 2 \mathrm{H}), 3.73(\mathrm{~s}, 3 \mathrm{H}) ;{ }^{13} \mathrm{C}$ NMR $\left(100 \mathrm{MHz}\right.$, DMSO-d $\left.d_{6}\right) \delta 180.45,156.69,132.72$, 126.03,113.76,55.71; FT-IR $\left(\mathrm{KBr}_{\mathrm{cm}} \mathrm{cm}^{-1}\right) 3312.45,3158.14,1635.19,1508.62,1489.55,1240.97,1031.78$, $829.78,509.71$.

\section{$\mathrm{N}$-(p-Tolyl)hydrazinecarbothioamide (6h)}

${ }^{1} \mathrm{H}$ NMR $\left(400 \mathrm{MHz}\right.$, DMSO- $\left.d_{6}\right) \delta 9.54(\mathrm{~s}, 1 \mathrm{H}), 9.04(\mathrm{~s}, 1 \mathrm{H}), 7.49(\mathrm{~d}, J=6.1 \mathrm{~Hz}, 2 \mathrm{H}), 7.10(\mathrm{~d}, J=8.1 \mathrm{~Hz}, 2 \mathrm{H})$, $4.76(\mathrm{~s}, 2 \mathrm{H}), 2.27(\mathrm{~s}, 3 \mathrm{H}) ;{ }^{13} \mathrm{C}$ NMR $\left(100 \mathrm{MHz}, \mathrm{DMSO}-d_{6}\right) \delta 180.09,137.18,133.68,128.99,124.02$, 20.96; FT-IR $\left(\mathrm{KBr}, \mathrm{cm}^{-1}\right)$ 3291.18, 3191.53, 1615.95, 1537.23, 1060.91, 806.74, 726.66, 441.48.

\section{Synthesis of target compound 7a-h}

4-(1-adamantyl)benzaldehyde $(1.0 \mathrm{mmol})$ and thiosemicarbazone $(6 \mathrm{a}-\mathrm{h})(1.2 \mathrm{mmol})$ were dissolved in ethanol $(6 \mathrm{~mL})$. Then two drops of acetic acid was added and the solution was refluxed for $3 \mathrm{~h}$.

\section{2-[4-(Adamantan-1-yl)benzylidene]-N-phenylhydrazine-1-carbothioamide (7a)}

Light yellow solid, yield $62 \%$, m.p. $178-180{ }^{\circ} \mathrm{C} ;{ }^{1} \mathrm{H}$ NMR (400 MHz, DMSO- $\left.d_{6}\right) \delta 11.91(\mathrm{~s}, 1 \mathrm{H}), 9.93(\mathrm{~s}, 1 \mathrm{H})$, $8.13(\mathrm{~s}, 1 \mathrm{H}), 7.80(\mathrm{~d}, J=8.3 \mathrm{~Hz}, 2 \mathrm{H}), 7.55(\mathrm{t}, J=7.5 \mathrm{~Hz}, 1 \mathrm{H}), 7.41(\mathrm{~d}, J=8.3 \mathrm{~Hz}, 2 \mathrm{H}), 7.26(\mathrm{dd}, J=24.5,9.3$ $\mathrm{Hz}, 3 \mathrm{H}), 2.06(\mathrm{~s}, 3 \mathrm{H}), 1.88(\mathrm{~s}, 6 \mathrm{H}), 1.74(\mathrm{~s}, 6 \mathrm{H}) .{ }^{13} \mathrm{C}$ NMR $\left(100 \mathrm{MHz}, \mathrm{DMSO}-d_{6}\right) \delta 127.92,125.50,124.39$, 55.35, 42.91, 36.65, 28.79. FT-IR $\left(\mathrm{KBr}, \mathrm{cm}^{-1}\right)$ 3298.94, 3141.85, 2899.12, 2846.27, 1621.21, 1545.86, 1221.97, 805.11,745.76; MS, m/z (\%): $408.3[\mathrm{M}+\mathrm{H}]^{+}$.

\section{2-[4-(Adamantan-1-yl)benzylidene]-N-(2-methoxyphenyl)hydrazine-1-carbothioamide (7b)}

Light yellow solid, yield $60 \%$ m.p. $187-189^{\circ} \mathrm{C} ;{ }^{1} \mathrm{H}$ NMR (400 MHz, DMSO- $\left.d_{6}\right) \delta 11.89(\mathrm{~s}, 1 \mathrm{H}), 10.00(\mathrm{~s}, 1 \mathrm{H})$, $8.36(\mathrm{~d}, J=7.3 \mathrm{~Hz}, 1 \mathrm{H}), 8.17(\mathrm{~d}, J=16.8 \mathrm{~Hz}, 1 \mathrm{H}), 7.72(\mathrm{~d}, J=8.3 \mathrm{~Hz}, 2 \mathrm{H}), 7.46(\mathrm{~d}, J=8.3 \mathrm{~Hz}, 2 \mathrm{H}), 7.24-$ 
$6.89(\mathrm{~m}, 3 \mathrm{H}), 3.91(\mathrm{~s}, 3 \mathrm{H}), 2.07(\mathrm{~s}, 3 \mathrm{H}), 1.90(\mathrm{~d}, J=12.0 \mathrm{~Hz}, 6 \mathrm{H}), 1.75(\mathrm{~s}, 6 \mathrm{H}) ;{ }^{13} \mathrm{C}$ NMR (100 MHz, DMSO$\left.d_{6}\right) \delta 151.41,143.06,127.53,125.78,123.50,120.35,56.56,42.86,39.44,36.60,28.74 ; \mathrm{FT}-\mathrm{IR}\left(\mathrm{KBr}, \mathrm{cm}^{-1}\right)$ $3154.70,2899.16,1599.45,1235.88,1193.39,1031.00,743.44 ; \mathrm{MS}, \mathrm{m} / \mathrm{z}(\%): 420.3[\mathrm{M}+\mathrm{H}]^{+}$.

\section{2-[4-(Adamantan-1-yl)benzylidene]-N-(3-methoxyphenyl)hydrazine-1-carbothioamide(7c)}

Light yellow solid, yield $79 \%$ m.p. $175-177^{\circ} \mathrm{C} ;{ }^{1} \mathrm{H}$ NMR (400 MHz, DMSO- $\left.d_{6}\right) \delta 11.89(\mathrm{~s}, 1 \mathrm{H}), 10.12$ (s, $1 \mathrm{H}), 8.15(\mathrm{~s}, 1 \mathrm{H}), 7.82(\mathrm{~d}, J=8.2 \mathrm{~Hz}, 2 \mathrm{H}), 7.63(\mathrm{~d}, J=11.3 \mathrm{~Hz}, 1 \mathrm{H}), 7.51-7.42(\mathrm{~m}, 2 \mathrm{H}), 7.40(\mathrm{~d}, J=10.2 \mathrm{~Hz}$, $1 \mathrm{H}), 7.03(\mathrm{t}, J=8.8 \mathrm{~Hz}, 1 \mathrm{H}), 2.07(\mathrm{~s}, 3 \mathrm{H}), 1.88(\mathrm{~s}, 6 \mathrm{H}), 1.75(\mathrm{~s}, 6 \mathrm{H}) ;{ }^{13} \mathrm{C}$ NMR $\left(100 \mathrm{MHz}, \mathrm{DMSO}-d_{6}\right) \delta$ $163.19,153.66,143.93,128.05,125.46,121.50,112.57$, 42.86, 36.60; FT-IR $\left(\mathrm{KBr}_{\mathrm{c}} \mathrm{cm}^{-1}\right)$ 3135.56, 2987.81, $2900.50,2847.06,1599.16,1541.58,1272.83,1064.02,548.94 ; M S, m / z(\%): 408.3[M+H]^{+}$.

\section{2-[4-(Adamantan-1-yl)benzylidene]-N-(4-methoxyphenyl)hydrazine-1-carbothioamide (7d)}

Light yellow solid, yield $53 \%$ m.p. $174-176{ }^{\circ} \mathrm{C} ;{ }^{1} \mathrm{H}$ NMR (400 MHz, DMSO- $\left.d_{6}\right) \delta 11.77(\mathrm{~s}, 1 \mathrm{H}), 9.99(\mathrm{~s}, 1 \mathrm{H})$, $8.14(\mathrm{~s}, 1 \mathrm{H}), 7.82(\mathrm{~d}, J=8.0 \mathrm{~Hz}, 2 \mathrm{H}), 7.41(\mathrm{~d}, J=8.0 \mathrm{~Hz}, 2 \mathrm{H}), 7.34-7.15(\mathrm{~m}, 3 \mathrm{H}), 6.78(\mathrm{~d}, J=7.9 \mathrm{~Hz}, 1 \mathrm{H})$, $3.77(\mathrm{~s}, 3 \mathrm{H}), 2.07(\mathrm{~s}, 3 \mathrm{H}), 1.88(\mathrm{~s}, 6 \mathrm{H}), 1.74(\mathrm{~s}, 6 \mathrm{H}) ;{ }^{13} \mathrm{C}$ NMR $\left(100 \mathrm{MHz}, \mathrm{DMSO}-d_{6}\right) \delta 159.49,143.52$, 129.18, 127.98, 125.46, 118.01, 111.53, 111.17, 55.64, 42.87, 36.60, 28.74; FT-IR $\left(\mathrm{KBr}, \mathrm{cm}^{-1}\right)$ 3125.97, 2899.24, 2845.76, 1597.70, 1546.41, 1463.44, 1288.53, 767.71, 546.23, 448.62; MS, m/z (\%): 420.3 $[\mathrm{M}+\mathrm{H}]^{+}$.

\section{2-[4-(Adamantan-1-yl)benzylidene]-N-(2-fluorophenyl)hydrazine-1-carbothioamide (7e)}

Light yellow solid, yield $49 \%$, m.p. $170-171{ }^{\circ} \mathrm{C} ;{ }^{1} \mathrm{H}$ NMR (400 MHz, DMSO- $\left.d_{6}\right) \delta 11.68(\mathrm{~s}, 1 \mathrm{H}), 9.95(\mathrm{~s}, 1 \mathrm{H})$, $8.11(\mathrm{~s}, 1 \mathrm{H}), 7.81(\mathrm{~d}, J=8.4 \mathrm{~Hz}, 2 \mathrm{H}), 7.40$ (d, J=8.6 Hz, 4H), $6.93(\mathrm{~d}, J=8.9 \mathrm{~Hz}, 2 \mathrm{H}), 5.75(\mathrm{~s}, 1 \mathrm{H}), 3.77$ (s, $3 \mathrm{H}), 2.06(\mathrm{~s}, 3 \mathrm{H}), 1.86(\mathrm{~d}, J=12.9 \mathrm{~Hz}, 6 \mathrm{H}), 1.74(\mathrm{~s}, 6 \mathrm{H}) ;{ }^{13} \mathrm{C} \mathrm{NMR}\left(100 \mathrm{MHz}, \mathrm{DMSO}-d_{6}\right) \delta 176.31,143.43$, 128.48, 127.96, 126.07, 125.44, 42.88, 36.62, 28.76; FT-IR (KBr, cm$\left.{ }^{-1}\right)$ 3138.58, 1198.23,803.73, 744.39, 493.02; MS, m/z (\%): $390.1[\mathrm{M}+\mathrm{H}]^{+}$.

\section{2-[4-(Adamantan-1-yl)benzylidene]-N-(3-fluorophenyl)hydrazine-1-carbothioamide (7f)}

Light yellow solid, yield $50 \%$ m.p. $188-190{ }^{\circ} \mathrm{C} ;{ }^{1} \mathrm{H}$ NMR (400 MHz, DMSO- $\left.d_{6}\right) \delta 11.79(\mathrm{~s}, 1 \mathrm{H}), 10.06(\mathrm{~s}, 1 \mathrm{H})$, $8.13(\mathrm{~s}, 1 \mathrm{H}), 7.82(\mathrm{~d}, J=8.3 \mathrm{~Hz}, 2 \mathrm{H}), 7.55$ (dd, $J=8.8,5.1 \mathrm{~Hz}, 2 \mathrm{H}), 7.41$ (d, $J=8.4 \mathrm{~Hz}, 2 \mathrm{H}), 7.20$ (t, $J=8.8$ $\mathrm{Hz}, 2 \mathrm{H}), 2.07$ (s, 3H), 1.89 (d, $J=9.0 \mathrm{~Hz}, 6 \mathrm{H}), 1.74(\mathrm{~s}, 6 \mathrm{H}) ;{ }^{13} \mathrm{C}$ NMR $\left(100 \mathrm{MHz}, \mathrm{DMSO}-d_{6}\right) \delta 176.72,158.89$, 143.56, 135.95, 128.49, 127.97, 125.44, 115.01, 42.87, 36.61, 28.75; FT-IR (KBr, cm $\left.{ }^{-1}\right)$ 3155.17, 2900.14, $2845.94,1526.22,1508.51,1196.02,928.48,836.46,553.47,499.03 ; \mathrm{MS}, \mathrm{m} / \mathrm{z}(\%): 408.3[\mathrm{M}+\mathrm{H}]^{+}$.

\section{2-[4-(Adamantan-1-yl)benzylidene]-N-(4-fluorophenyl)hydrazine-1-carbothioamide (7g)}

Light yellow solid, yield $57 \%$, m.p. $177-179{ }^{\circ} \mathrm{C} ;{ }^{1} \mathrm{H}$ NMR (400 MHz,DMSO- $\left.d_{6}\right) \delta 11.68(\mathrm{~s}, 1 \mathrm{H}), 9.95(\mathrm{~s}, 1 \mathrm{H})$, $8.11(\mathrm{~s}, 1 \mathrm{H}), 7.80(\mathrm{~s}, 2 \mathrm{H}), 7.41$ (s, 4H), 6.94 (s, 2H), 3.77 (s, 3H); ${ }^{13} \mathrm{C}$ NMR (100 MHz, DMSO-d $\left.d_{6}\right) \delta 157.42$, 
143.23, 131.91, 127.90, 125.41, 113.78, 55.77, 42.92, 36.64, 28.78; FT-IR $\left(\mathrm{KBr}, \mathrm{cm}^{-1}\right) 3135.42,2980.90$, $2899.64,1608.72,1532.53,1510.61,1233.24,1014.33,804.52,548.38 ; \mathrm{MS}, \mathrm{m} / \mathrm{z}(\%): 420.3[\mathrm{M}+\mathrm{H}]^{+}$.

\section{2-[4-(Adamantan-1-yl)benzylidene]-N-(p-tolyl)hydrazine-1-carbothioamide (7h)}

Light yellow solid, yield $89 \%$, m.p. $196-197{ }^{\circ} \mathrm{C} ;{ }^{1} \mathrm{H}$ NMR $\left(400 \mathrm{MHz}, \mathrm{DMSO}-d_{6}\right) \delta 11.71(\mathrm{~s}, 1 \mathrm{H}), 9.97(\mathrm{~s}, 1 \mathrm{H})$, $8.12(\mathrm{~s}, 1 \mathrm{H}), 7.81(\mathrm{~d}, J=8.2 \mathrm{~Hz}, 2 \mathrm{H}), 7.42(\mathrm{t}, J=9.5 \mathrm{~Hz}, 4 \mathrm{H}), 7.17(\mathrm{~d}, J=8.0 \mathrm{~Hz}, 2 \mathrm{H}), 2.31(\mathrm{~s}, 3 \mathrm{H}), 2.06(\mathrm{~s}$, $3 \mathrm{H}), 1.88(\mathrm{~s}, 6 \mathrm{H}), 1.74(\mathrm{~s}, 6 \mathrm{H}) ;{ }^{13} \mathrm{C}$ NMR $\left(100 \mathrm{MHz}, \mathrm{DMSO}-d_{6}\right) \delta 143.30,131.86,128.96,127.93,126.09$, 125.44, 42.87, 36.6, 28.75; FT-IR (KBr, cm$\left.{ }^{-1}\right) 3132.79,2896.53,2845.57,1532.84,1492.85,1264.08$, 1201.14, 1176.71, 835.84, 449.53; MS, m/z (\%): $404.4[\mathrm{M}+\mathrm{H}]^{+}$.

\section{In vitro antibacterial activity evaluation}

Minimum Inhibitory Concentration (MIC) assays using standard microdilution methods were carried out in 96-well microplates based on a modified procedure described previously according to the guidelines of the Clinical and Laboratory Standards Institute [30-31]. All compounds were prepared as $5 \mathrm{mg} / \mathrm{mL}$ solutions in dimethyl sulfoxide (DMSO) and were tested in a final concentration range of $1-8 \mu \mathrm{g} / \mathrm{mL}$. MICs for the reference antibiotic Ampicillin against quality control strains were used to confirm the validity of the screen.

All experiments were performed in duplicate and repeated three times.

\section{Declarations}

Acknowledgements We thank Liwen Bianji, Edanz Group China (www.liwenbianji.cn/ac), for editing the English text of a draft of this manuscript.

Authors' contributions Jiahui Zhu and Yan Xia performed all experiments, purified all compounds, analyzed the data and summarized the results. Jiahui Zhu tested all compounds for their antibacterial activity. Dongfeng Li and Guosheng Teng helped in the compiling the data of the manuscript. Yan Xia and Ruibin Hou conceived and designed this research and wrote the manuscript. All authors have contributed to the final version and approved the final manuscript.

Funding This work was financially supported by the National Natural Science Foundation of China (21502008) and Natural Science Foundation of Jilin Province (00005005031).

\section{Compliance with ethical standards}

Conflicts of interest All the authors declared no conflict of interest.

Author Information

\section{Affiliations}


Jiahui Zhu ${ }^{1,2} \cdot$ Guosheng Teng ${ }^{1} \cdot$ Dongfeng $\mathrm{Li}^{1} \cdot$ Ruibin $\mathrm{Hou}^{1,2} \cdot$ Yan Xia ${ }^{1,2}$

Yan Xia

Email: hmz20130521@163.com

Ruibin Hou

Email: hrb1018@163.com

${ }^{1}$ College of Chemistry and Life Science, Changchun University of Technology, Changchun 130012, China

${ }^{2}$ Advanced Institute of Materials Science, Changchun University of Technology, Changchun 130012, China

\section{References}

1. Ventola CL (2015) The Antibiotic Resistance Crisis Part 1 Causes and Threats. Pharmacol Ther 40 : 277-283. https://doi.org/

2. Brooks BD (2014) Therapeutic strategies to combat antibiotic resistance. Adv Dru Deliv Rev 78: 1427. https://doi.org/10.1016/j.addr.2014.10.027

3. Livermore DM (2004) The need for new antibiotics. Clin Microbiol Infect 10: 1-9. https://doi.org/10.1111/j.1465-0691.2004.1004.x

4. Puertoa SA, Fernandeza GJ, Castillo LDJ, Pino SJM, Angulo PG (2006) In vitro activity of beta-lactam and non-beta-lactam antibiotics in extended-spectrum beta-lactamase-producing clinical isolates of Escherichia coli. Diagn Microbiol Infect Dis 54: 135-139. https://doi.org/10.1016/j.diagmicrobio.2005.08.018

5. Krishnanjaneyulu IS, Saravanan G, Vamsi J, Supriya P, Bhavana JU, Sunil Kumar MV (2014) Synthesis, characterization and antimicrobial activity of some novel benzimidazole derivatives. $\mathrm{J}$ Adv Pharm Technol Res 5: 21-27. https://doi.org/10.4103/2231-4040.126983

6. Moellering Jr RC (2011) Discovering new antimicrobial agents. Int J Antimicrob Agents 2011; 37: 2-9. https://doi.org/10.1016/j.ijantimicag.2010.08.018

7. De Araújo Neto LN, De Lima M do CA, De Oliveira, JF, et al (2020) Thiophene-thiosemicarbazone derivative (L10) exerts antifungal activity mediated by oxidative stress and apoptosis in C. albicans. Chem Biol Interact 320: 109028. https://doi.org/10.1016/j.cbi.2020.109028

8. Khan SA, Kumar P, Joshi R, Iqbal PF, Saleem K (2008) Synthesis and in vitro antibacterial activity of new steroidal thiosemicarbazone derivatives. Eur J Med Chem 43: 2029-2034. https://doi.org/ 10.1016/j.ejmech.2007.12.004

9. Matsa R, Makam P, Kaushik M, Hoti SL, Kannan T (2019) Thiosemicarbazone derivatives: Design, synthesis and in vitro antimalarial activity studies. Eur J Pharm Sci 137: 104986. https://doi.org/10.1016/j.ejps.2019.104986 
10. Zhang X, Qi F, Wang S, Song J, Huang J (2020) Synthesis, structure, in silico ADME evaluation and in vitro antioxidant of (E)-N-(4-ethylphenyl)-2-(isomeric methylbenzylidene) thiosemicarbazone derivatives. J Mol Struct 1199: 126972. https://doi.org/10.1016/j.molstruc.2019.126972

11. Trotsko N, Golus J, Kazimierczak P, Paneth A, Przekora A, Ginalska G, Wujec M (2020) Design, synthesis and antimycobacterial activity of thiazolidine-2,4-dione-based thiosemicarbazone derivatives. Bioorg Chem 97: 103676. https://doi.org/10.1016/j.bioorg.2020.103676

12. He Z, Qiao H, Yang F, Zhou W, Gong Y, Zhang X, Wang H, Zhao B, Ma L, Liu HM, Zhao W (2019) Novel thiosemicarbazone derivatives containing indole fragment as potent and selective anticancer agent. Eur J Med Chem 184: 111764. https://doi.org/10.1016/j.ejmech.2019.111764

13. Pham VH, Phan TPD, Phan DC, Vu BD (2020) Synthesis and Bioactivity of Thiosemicarbazones Containing Adamantane Skeletons. Molecules 25: 324. https://doi.org/10.3390/molecules25020324

14. Siddiqui EJ, Azad I, Khan AR, Khan T (2019) Thiosemicarbazone Complexes as Versatile Medicinal Chemistry Agents: A Review. J Drug Deliv Ther 9: 689-703. https://doi.org/10.22270/jddt.v9i3.2888

15. Pervez H, Iqbal MS, Tahir MY, Choudhary MI, Khan KM (2007) Synthesis of some $\mathrm{N}^{4}$-substituted isatin-3-thiosemicarbazones. Nat Prod Res 21: 1178-1186. https://doi.org/10.1080/14786410601129770

16. Basarić N, Sohora M, Cindro N, Mlinarić-Majerski K, De Clercq E, Balzarini J (2014) Antiproliferative and antiviral activity of three libraries of adamantane derivatives. Arch Pharm 2014; 347: 334-340. https://doi.org/10.1002/ardp.201300345

17. Fesatidou M, Zagaliotis $P$, Camoutsis $C$, et al (2018) 5-Adamantan thiadiazole-based thiazolidinones as antimicrobial agents. Design, synthesis, molecular docking and evaluation. Bioorg Med Chem 26: 4664-4676. https://doi.org/10.1016/j.bmc.2018.08.004

18. Anusha S, Mohan CD, Ananda H, et al (2016) Adamantyl-tethered-biphenylic compounds induce apoptosis in cancer cells by targeting Bcl homologs. Bioorg Med Chem Lett 26: 1056-1060. https://doi.org/10.1016/j.bmcl.2015.12.026

19. Göktaş F, Vanderlinden E, Naesens L, Cesur N, Cesur Z (2012) Microwave assisted synthesis and antiinfluenza virus activity of 1-adamantyl substituted N-(1-thia-4-azaspiro[4.5]decan-4-yl)carboxamide derivatives. Bioorg Med Chem 20: 7155-7159. https://doi.org/10.1016/j.bmc.2012.09.064

20. Fytas C, Zoidis G, Tsotinis A, Fytas G, Khan MA, Akhtar S, Rahman KM, Thurston DE (2015) Novel 1(2-aryl-2-adamantyl)piperazine derivatives with antiproliferative activity. Eur J Med Chem 93: 281290. https://doi.org/10.1016/j.ejmech.2015.02.021

21. Aguiar DF, Dutra LLA, Dantas WM, et al (2019) Synthesis, Antitumor and Cytotoxic Activity of New Adamantyl 0-Acylamidoximes and 3-Aryl-5-Adamantane-1, 2, 4-0xadiazole Derivatives. Chem Sel 4: 9112-9118. https://doi.org/10.1002/slct.201901285

22. Tsuzuki N, Hama T, Kawada M, Hasui A, Konishi R, Shiwa S, Ochi Y, Futaki S, Kitagawa K (1994) Adamantane as a brain-directed drug carrier for poorly absorbed drug. 2. AZT derivatives conjugated with the 1-adamantane moiety. J Pharm Sci 83: 481-484. https://doi.org/10.1002/jps.2600830407 
23. Gerzon K, Krumkalns EV, Brindle RL, Marshall FJ, Root MA (1963) The adamantyl group in medicinal agents. I. Hypoglycemic $N$-arylsulfonyl- $N$ '-adamantylureas. J Med Chem 6: 760-763. https://doi.org/10.1021/jm00342a029

24. Rapala RT, Kraay RJ, Gerzon K (1965) The adamantyl group in medicinal agents. II. Anabolic steroid 17-beta-adamantoates. J Med Chem 8: 580-583. https://doi.org/10.1021/jm00329a007.

25. Gerzon K, Kau D (1967) The adamantyl group in medicinal agents. 3. Nucleoside 5'-adamantoates. The adamantoyl function as a protecting group. J Med Chem 10: 189-199. https://doi.org/10.1021/jm00314a014

26. Martin YC, Kofron JL, Traphagen LM (2002) Do structurally similar molecules have similar biological activity? J Med Chem 45: 4350-4358. https://doi.org/10.1021/jm020155c

27. Bender A, Jenkins JL, Scheiber J, Sukuru SCK, Glick M, Davies J (2009) How similar are similarity searching methods? A principal component analysis of molecular descriptor space. J Chem Inf Model 49: 108-119. https://doi.org/10.1021/ci800249s

28. Fesatidou M, Zagaliotis P, Camoutsis Ch, et al (2018) 5-Adamantan thiadiazole-based thiazolidinones as antimicrobial agents. Design, synthesis, molecular docking and evaluation. Bioorg Med Chem 26: 4664-4676. https://doi.org/10.1016/j.bmc.2018.08.004

29. Davis WW, Stout TR (1971) Disc plate method of microbiological antibiotic assay. II. Novel procedure offering improved accuracy. Appl Microbiol 22: 666-670.

30. Valentine SC, Contreras D, Tan S, Real LJ, Chu S, Xu HH (2008) Phenotypic and molecular characterization of Acinetobacter baumannii clinical isolates from nosocomial outbreaks in Los Angeles County, California. J Clin Microbiol 46: 2499-2507. https://doi.org/10.1128/JCM.00367-08

31. CLSI. Methods for dilution antimicrobial susceptibility tests for bacteria that grow aerobically; Approved Standard-Ninth Edition. M07-A9. Clinical and Laboratory Standards Institute, Wayne, PA, 2012.

\section{Figures}




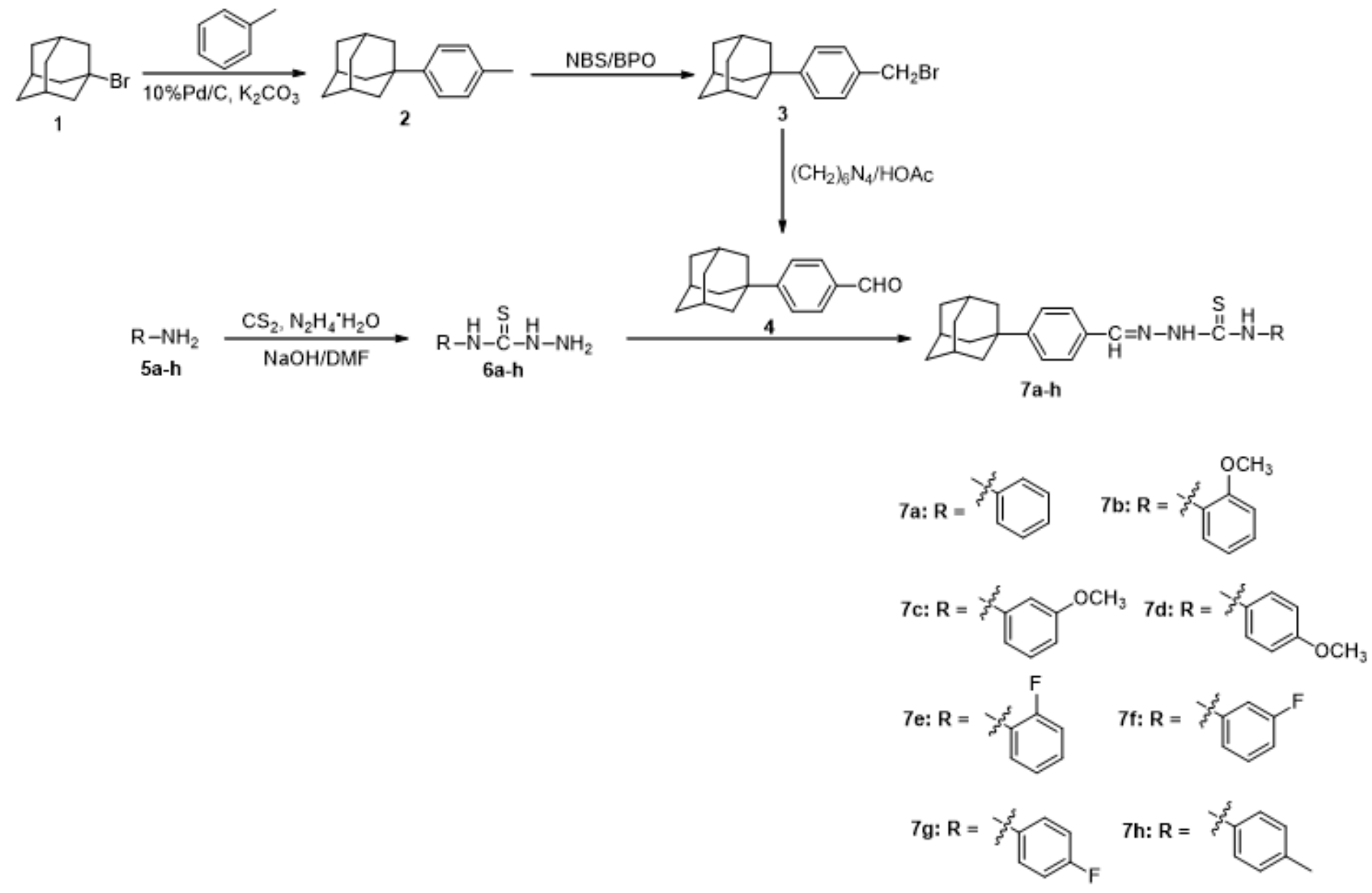

Figure 1

Synthetic procedures of compounds 7a-h. 